\title{
PIEZOELECTRIC EFFECT IN STRAINED CdTe-BASED HETEROSTRUCTURES
}

\author{
J. Cibert, R. ANdré and Le Si Dang \\ Laboratoire de Spectrométrie Physique, CNRS et Université J. Fourier-Grenoble \\ BP87, 38402 St Martin d'Hères, Cedex, France
}

Strained III-V or II-VI heterostructures grown along a polar axis incorporate a piezoelectric field which strongly alters their electronic properties. We review some experimental studies of this built-in field, and its consequences, in $\mathrm{CdTe}-\mathrm{CdMnTe}$ and $\mathrm{CdTe}-\mathrm{CdZnTe}$ heterostructures. This includes: (i) the measure of the built-in field, with as an example the dependence of the piezoelectric coefficient on bond length; (ii) the description of the exciton formed by an electron and a hole which can be continuously separated in space by the piezoelectric field; (iii) some examples of the extreme sensitivity to any additional electric field.

PACS numbers: $77.65 . \mathrm{Bn}, 78.20 . \mathrm{Hp}, 78.66 . \mathrm{Hf}$

\section{Introduction}

The zinc-blende structure of most usual semiconductor compounds, as opposed to the diamond structure of elemental semiconductors, has two different atoms occupying the two sites of the unit cell, and hence lacks inversion symmetry. As a result these materials exhibit piezoelectric effect: applying a strain induces an electric polarization, with the piezoelectric tensor relating the polarization to the strain $[1,2]$. As a matter of fact, zinc-blende semiconductors are among the simplest materials with piezoelectric properties (the piezoelectric tensor has only one independent parameter, $e_{14}$ ) and have been considered for a long time as model structures to check theoretical calculations of the piezoelectric effect [3]. However the magnitude of the strain-induced polarization is usually small, and in bulk materials it is easily screened by charge transfer. Recently the domain has been renewed for two very different reasons: on the one hand a new method of calculating the piezoelectric effect [4] and on the other hand the idea that it can play a very significant role in strained heterostructures grown along a polar axis [5], where the piezoelectric field may be quite large (due to the possibility to incorporate large trigonal strains) and is less easily screened by mobile charges. In such heterostructures the piezoelectric field gives rise to a built-in quantum confined Stark effect, greatly altering their electronic and optical properties, with in particular a strongly enhanced sensitivity to additional electric fields of any origin. 
In this paper we review some properties of piezoelectricity in quantum wells, as they have been studied in the case of CdTe heterostructures. We first briefly recall the mechanisms at the origin of piezoelectric eflect in zinc-blende semiconductors, and the results of recent calculations; then we show that the study of properly designed heterostructures allows one to measure optically the built-in field; as an example, a strong variation of the piezoelectric coefficient in $\mathrm{CdTe}$ is found when heterostructures are studied under hydrostatic pressure, in agreement with theoretical predictions by Dal Corso et al. [10]. Then we describe how the exciton is modified in piezoelectric quantum wells. Finally we review some consequences of the linear Stark effect due to biasing by the built-in field.

\section{Piezoelectric effect in zinc-blende semiconductors}

The usual description of a strained material corresponds to the macroscopic strain, described by the strain tensor, related to the stress through the elastic constants $c_{i j}$. In a material with cubic symmetry a given strain can be decomposed into an isotropic strain (associated to $c_{11}+2 c_{12}$ ), tetragonal strain components (associated to $c_{11}-c_{12}$ ) and trigonal strain components (associated to $c_{44}$ ). Only the trigonal strain components are piezoelectrically active, inducing a polarization $P_{x}=e_{14} \varepsilon_{y z}$, etc.

At the microscopic level the above (macroscopic) strain tensor describes only the deformation of the unit cell: in addition, if the unit cell includes more than one atom, one has to consider the so-called internal strain, which describes the relative motion of atoms within the unit cell. In the zinc-blende structure the macroscopic strain describes the relative motion of one type of atoms, within one sublattice, and the internal strain describes the motion of one type of atom relatively to atoms of the other type. In other words, the macroscopic strain describes the deformation of the corners of the basic tetrahedron, while the internal strain describes the motion of the central atom within the tetrahedron.

For a uniform strain, the internal strain is related to the macroscopic strain through the Kleinman parameter $\zeta[6]$, which is defined as follows. If $\zeta=0$, the atom remains at the centre of the deformed tetrahedron; if only bond-bending is allowed, $\zeta=1$ (then the internal strain is maximum). Experimental values of $\zeta$ are scarce, of the order of $0.7-0.8$ for $\mathrm{Si}$ and GaAs [7]. Theoretical estimates are difficult. The simplest models are the valence force field models, with only nearest-neighbours interactions $[8,9]$. Only qualitative estimates are expected, but the model of Ref. [9] gives

$$
\zeta=\frac{C_{0}-4 C_{1}}{C_{0}+8 C_{1}}
$$

where $C_{0}$ is the bond stretching energy and $C_{1}$ the bond bending energy. As the same model relates $C_{0}$ to $c_{11}+2 c_{12}$ and $C_{1}$ to $c_{11}-c_{12}$, the final result is

$$
\zeta=\frac{1-\frac{2}{3} \frac{c_{11}-c_{12}}{c_{11}+2 c_{12}}}{1+\frac{4}{3} \frac{c_{11}-c_{12}}{c_{11}+2 c_{12}}} .
$$


An optical phonon at the centre of the Brillouin zone corresponds to a pure internal strain, with zero macroscopic strain. From the polarization induced by such a phonon (which is measured in the TO-LO splitting), one deduces the Born effective charge $Z^{*}$ (in electron charge unit $e$ ). In CdTe the experimental value is $Z^{*}=2.35$. From geometrical considerations one obtains the piezoelectric coefficient corresponding to this internal strain (i.e., corresponding to the motion of the two ions with the charges $\pm Z^{*} e$ ),

$$
e_{14}^{\text {ion }}=\frac{Z^{*} e}{a_{0}^{2}} \zeta
$$

The actual piezoelectric coefficient is calculated through more elaborate theories [4] as

$$
e_{14}=\frac{Z^{*} e}{a_{0}^{2}} \zeta+e_{14}^{\text {electron }}
$$

where the "electronic contribution" $e_{14}^{\text {electron }}$ is calculated at vanishing internal strain. It appears $[3,4]$ that the two contributions are opposite in sign; experiments and theories generally agree on the point that in III-V compounds the electronic contribution is slightly larger and the resulting piezoelectric coefficient is negative (i.e., an elongation along the [111] axis induces an electric field pointing from the type-V to the type-III atom), while in most II-VI compounds the electronic contribution is smaller and the piezoelectric coefficient is positive; in several compounds the two contributions are close to cancel and the piezoelectric coefficient is small: this is the case for InAs, $\mathrm{ZnTe}, \mathrm{ZnSe}$ and CdTe. In this case, Dal Corso et al. [10] have shown that the value of the piezoelectric coefficient is likely to be changed by a reasonably large strain, i.e., the piezoelectric effect is expected to be non-linear. We shall come back to this point and show that this non-linearity can be measured in heterostructures under hydrostatic pressure.

\section{The measure of the built-in field in strained heterostructures}

Piezoelectric effects have been demonstrated in several systems with the wurtzite structure (CdS-CdSe [11]) or the zinc-blende structure (GaAs-GaInAs [12], GaSb-AISb [13], CdTe-CdZnTe [14], CdTe-CdMnTe [15], InAs-GaSb [16]...). In strained heterostructures of semiconductors with the zinc-blende structure the growth axis is generally [111] or [112]. To increase the optical density (see below) many studies have been done on superlattices: then the strain state is poorly known (somewhere between free-standing and coherent on the buffer layer). In some studies of the GaAs-GaInAs system a single GaInAs quantum well is introduced in a GaAs $p$ - $i-n$ structure, allowing to apply an additional electric field [17] and to measure the built-in field [18].

A fully optical measure of the built-in field is also possible. To this purpose we grow samples featuring on a thick $\mathrm{Cd}_{1-x} \mathrm{Zn}_{x}$ Te or $\mathrm{Cd}_{1-x} \mathrm{Mn}_{x}$ Te buffer layer several CdTe quantum wells of different thickness $L_{\mathrm{w}}$, with thick barriers of the same composition as the buffer layer. The buffer layer is thick enough to be practically strain-free (as checked by X-ray diffraction), hence the barriers are cubic and only the quantum wells are strained, with the same strain in all quantum wells. 
The piezoelectric field in the quantum wells is easily calculated from the lattice mismatch $\delta a / a$; for the [111] growth direction we have

$$
F=-\frac{2 \sqrt{3}}{\varepsilon \varepsilon_{0}} \frac{c_{11}+2 c_{12}}{c_{11}+2 c_{12}+4 c_{44}} e_{14} \frac{\delta a}{a} .
$$

For other directions straightforward but sometimes tedious calculations give smaller induced fields $[19,20]$ (note that other calculations have been published whose results agree with these references for directions of high symmetry, but not for arbitrary directions). Two directions are particularly singular:

- for growth on (001) the strain tensor has only isotropic and tetragonal components and the polarization is zero;

- for growth on (110) the induced polarization is within the growth plane and should be as easily screened as in bulk materials.

The potential profile of such a strained quantum well, with unstrained barriers, comprises the band offset between the barrier and well materials, including strain effects (deformation potentials), and the linear potential due to the field. The calculation of energy levels is straightforward [14,15]; for the optical transition between the first confined electron and heavy hole levels one obtains

$$
E\left(e_{1} h_{1}\right)=E_{\mathrm{G}}+E_{\mathrm{el}}+E_{\mathrm{hl}}-e F L_{\mathrm{w}}-E_{\mathbf{x}},
$$

where $E_{\mathrm{G}}$ is the bandgap of strained $\mathrm{CdTe}, E_{\mathrm{x}}$ is the exciton binding energy (see below), and $E_{\mathrm{el}}$ and $E_{\mathrm{hl}}$ are confinement energies: for wide enough quantum wells the carriers are confined in triangular wells in the two opposite corners of the quantum well, and do not see the respective opposite barrier, so that the confinement energies are constant. The result is that the $e_{1} h_{1}$ energy shifts to the red linearly with the quantum well thickness $L_{\mathrm{w}}$. This is exemplified in Fig. 1 for a $\mathrm{CdTe}-\mathrm{CdMnTe}$ sample. On such a plot the slope directly gives the built-in field,
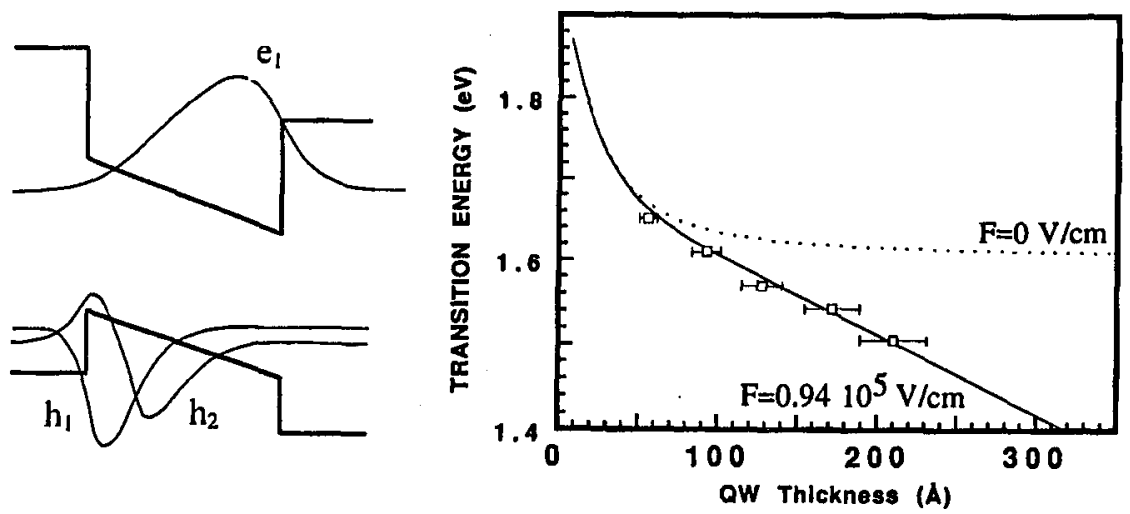

Fig. 1. Schematic potential profile of a piezoelectric quantum well (left) and plot of the $e_{1} h_{1}$ energy vs. well thickness in $\mathrm{CdTe}-\mathrm{Cd}_{0.82} \mathrm{Mn}_{0.18} \mathrm{Te}$ (solid line: calculated with a built-in field; dashed line: without field; squares: experimental). 


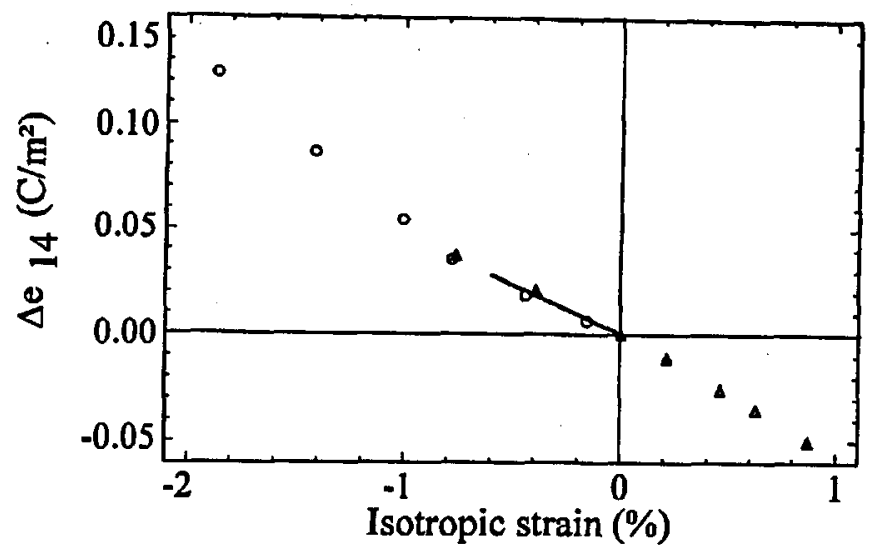

Fig. 2. Variation of the piezoelectric coefficient (closed symbols: experimental; open symbols: calculated in Ref. [10]; solid line: valence force field estimation).

which can be measured as a function of the lattice mismatch using samples with different barrier compositions [21, 22]: unexpectedly the measured field somewhat differs from the value calculated from Eq. (5) and the published value of $e_{14}$ in bulk CdTe.

Part of the discrepancy can be explained by the non-linear character of the piezoelectric effect, i.e., the piezoelectric coefficient is not constant but depends on the isotropic strain (Fig. 2). This effect is experimentally demonstrated by applying a hydrostatic pressure to a sample with several quantum wells (thus changing the isotropic strain component but not the trigonal component), and once again plotting the $e_{1} h_{1}$ energy as a function of the quantum well thickness for different values of the pressure. The slope (i.e., the field, i.e., the piezoelectric coefficient) increases as much as a factor of two for a pressure $2.5 \mathrm{GPa}$ [23]. The experimental variation of the piezoelectric coefficient remarkably agrees with the variation calculated in Ref. [10], which attributes the effect mainly to an increase in the internal strain parameter $\zeta$ due to the increase in the isotropic strain component (with only minor changes of the Born charge $Z^{*}$ and of the electronic contribution $e_{14}^{\text {electron, }}$ and no dependence of $e_{14}$ on the trigonal strain). Actually this variation of $\zeta$ is expected in the qualitative description by the valence force field model, Eq. (2): under hydrostatic pressure the bulk modulus $\left(c_{11}+2 c_{12}\right) / 3$ increases; moreover the shear elastic coefficient $\left(c_{11}-c_{12}\right)$ decreases as the phase transition from the zinc-blende to the rocksalt structure is approached (it occurs at $3.3 \mathrm{GPa}$ in $\mathrm{CdTe}$ [24]). Both effects tend to increase the internal strain, in agreement with the fact that it is maximum if bond bending is much easier than bond stretching.

Similar non-linearities should be observed in other materials where the two contributions to the piezoelectric coefficient are nearly cancelled. Note that an increase in the internal strain tends to increase the absolute value of $e_{14}$ if the ionic contribution is dominant (as in CdTe and other II-VI compounds) but should decrease it if the electronic contribution is dominant (as in GaInAs). 


\section{Oscillator strength and excitons in piezoelectric quantum wells}

In a piezoelectric quantum well made of a type-I system (such as CdTe$\mathrm{CdZnTe}, \mathrm{CdTe}-\mathrm{CdMnTe}, \mathrm{GaInAs}-\mathrm{GaAs})$ the first confined levels $\left(e_{1}\right.$ and $\left.h_{1}\right)$ progressively separate in space as the quantum well thickness $L_{\mathrm{w}}$ increases: this can be viewed as a continuous tuning from a type-I configuration (electron and hole wave functions peaked at the same place) to a type-II configuration (decreased electron-hole overlap). Hence the oscillator strength, which is a function of the overlap, decreases. On the other hand the second confined level, if it exists, is less confined towards the barrier: not only the $e_{1} h_{2}$ transition is not forbidden (as it is in a perfect square quantum well due to symmetry), but for certain quantum well widths it can be stronger than the $e_{1} h_{1}$ transition [25].

However this is not the whole story and in II-VI compounds excitons play an important role. The properties of the excitons were studied using a variational approach. In non-piezoelectric quantum wells a one-parameter calculation is generally very efficient. In the present case we used [26] a two-parameter calculation, which proved to give accurate results not only for the exciton binding energy (which is easy), but also for the wave function, as checked through the value of the oscillator strength. More precisely the trial envelope function was

$$
\frac{1}{\sqrt{N}} \exp \left[-\frac{\sqrt{x_{\mathrm{eh}}^{2}+y_{\mathrm{eh}}^{2}+\alpha z_{\mathrm{eh}}^{2}}}{a_{x}}\right] \psi_{\mathrm{e}}\left(z_{\mathrm{e}}\right) \psi_{\mathrm{h}}\left(z_{\mathrm{h}}\right),
$$

where the $\psi$ 's are the wave functions of single carriers confined in the quantum well, $x_{\mathrm{eh}}, y_{\mathrm{eh}}$ and $z_{\mathrm{eh}}$ are the electron-hole relative coordinates, $z$ being the growth direction. The normalizing factor $N$ is essential for the calculation of the oscillator strength, proportional to $\left|\left\langle\psi_{\mathrm{e}} \mid \psi_{\mathrm{h}}\right\rangle\right|^{2} / N$. The variational parameters are $\alpha$ and $a_{x}$.

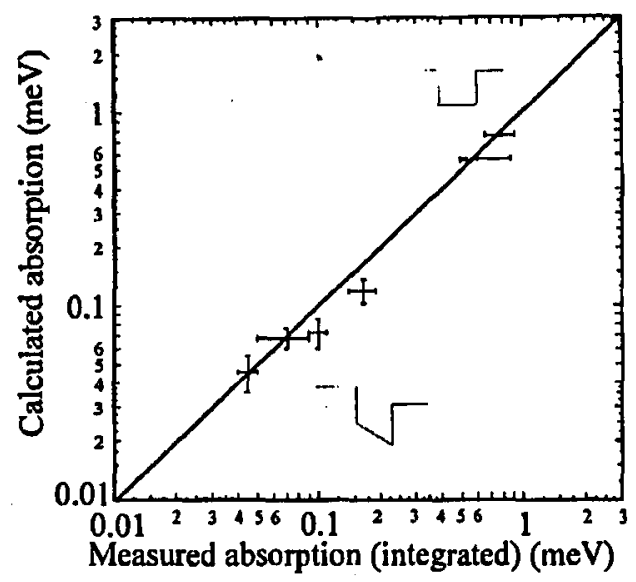

Fig. 3. Calculated and measured absorption by piezoelectric (four samples on the left) and square (three samples on the right) quantum wells. 
Results [26] can be summarized as follows, for wide quantum wells:

(i) The exciton binding energy decreases, approximately as $e^{2} / 4 \pi \varepsilon \varepsilon_{0} L_{\mathrm{w}}$. This is easily understood since electron and hole are pushed away toward the opposite barriers by the build-in field, and maintained by the quantum-well potential at the relative distance $L_{\mathrm{w}}$, with precisely this Coulomb energy.

(ii) The Bohr radius within the growth plane, $a_{x}$, increases approximately as $L_{\mathrm{w}}$. Actually increasing $a_{x}$ up to $\approx L_{\mathrm{w}}$ reduces the "kinetic" energy (which varies as $1 / a_{x}^{2}$ and becomes definitely smaller than the Coulombic energy) but increasing it further would begin to reduce the gain in the Coulomb term.

(iii) The dimensionality parameter $\alpha$ decreases, i.e., the two-dimensional character of the exciton increases: this means that for wide wells, the exciton is weak enough that it no more influences the motion in the $z$-direction.

One consequence is that the oscillator strength decreases faster than the overlap of the single-carrier wave functions: there is a multiplicative factor, $1 / N$, which varies as $1 / L_{\mathrm{w}}^{2}$ for wide enough quantum wells, but is obtained more precisely in the variational calculation. Calculated values are compared to experimental results in Fig. 3; the agreement is remarkable since the calculation involves no adjustable parameter.

\section{High sensitivity to additional electric fields}

Applying an electric field along the normal of a usual square quantum well induces the so-called quantum confined Stark effect. Due to symmetry, in a square quantum well first-order terms vanish and the shift of the energy levels is quadratic in field. In a piezoelectric quantum well of course a linear shift is expected: the actual field in the quantum well is the sum of the built-in field and the piezoelectric field. This picture has been experimentally checked in GaAs-GaInAs quantum wells inserted in a $p-i-n$ diode, which gives a direct evidence of the existence of the built-in field [17], and a method to measure it [18].

Applying a well controlled electric field across a II-VI heterostructure is still difficult. We have applied a field on a (111) CdTe-CdZnTe quantum well (with strain in CdTe only) to check that the piezoelectric field is in the Cd-to-Te direction, i.e., the piezoelectric coefficient is positive. But it was not possible to measure it.

However the high sensitivity to an additional field or to space charges which results from biasing by the built-in piezoelectric field has other important consequences and we shall give a few examples now.

\subsection{Doping}

Bassani et al. [27] have inserted thin layers of In (a donor impurity in CdTe) at different positions in (211) CdTe-CdZnTe quantum wells. A nominally undoped sample was grown under the same conditions. Samples were characterized by photoluminescence. The measured fields were identical in the undoped sample and in the sample $\delta$-doped in the substrate-side parts of the wells. It was strongly screened however in the sample doped in the surface-side parts of the wells. Hence 
the donor level is at higher energy than the quantum well level, if the donor is on the surface-side of the well, while it is at lower energy if the donor sits on the substrate side. This is a direct evidence that the field points toward the surface, which was, as in most CdTe samples grown by molecular beam epitaxy, the Te-rich surface (the B-surface). This confirms that the piezoelectric coefficient is positive in such samples.

Similar screening was also observed in modulation-doped samples (donors in the barrier). If one compares piezoelectric modulation-doped quantum wells to non-piezoelectric ones, a more efficient transfer is expected into the quantum well from the barrier which is raised in energy by the built-in field [28]. Moreover the field drives the electrons away towards the opposite interface. The interesting effect should be to reduce impurity scattering in the two-dimensional gas. But also it induces a compensating electric field which could alter the field measurements at low mismatch.

\subsection{Band bending: surface effects}

Fermi-level pinning at the surface of the sample, and the resulting band bending, have been detected as a sizable shift of the transition energy of a quantum well close to the surface, with respect to that of deeply buried wells [21].

\subsection{Field fluctuations and linewidth}

In non-piezoelectric quantum wells the linewidth generally decreases for increasing well thickness, and is attributed to thickness fluctuations due to interface roughness. In piezoelectric wells on the contrary it often increases linearly $[11,29]$, with a slope which is strongly correlated with the width of the X-ray diffraction peak. Then the broadening must be attributed to fluctuations of the built-in field. A model of microscopic strain fluctuations [29] was used to calculate, on one hand the resulting field fluctuation which broadens the optical transition, and on the other hand the longitudinal broadening of the Bragg peak as it appears in a map of the reciprocal space in the $\theta-2 \theta$ scan.

\subsection{Non-linear optics}

The strong initial interest in piezoelectric structures arised because of the expected non-linear behaviour when a reasonably large density of photocreated electron-hole pairs screen the built-in field. The expected blue-shift was detected quite early in several systems $[30,11,13,15]$ using $\mathrm{cw}$ spectroscopy. However it appears now that the decay times involved may be very long. In the case of CdS-CdSe this is expected since the band alignment is type II, and a detailed study has been published [30]. In GaAs-GaInAs more recent studies attribute the blue shift essentially to charge transfer on a long distance to screen the field over the entire heterostructure [31]. In CdTe-CdZnTe and CdTe-CdMnTe both local screening, within the quantum well, and large distance screening, with longer decay times, have been detected [19, 32]. Time-resolved spectroscopy, and a detailed 
model taking into account the equilibrium between excitons and free carriers, support the existence of the first mechanism.

\section{Acknowledgments}

This work was completed within the CNRS-CEA group "Microstructures de Semiconducteurs II-VI". In particular the name of many collaborators, who participated only to parts of this work, can be found in the references.

\section{References}

[1] W.G. Cady, Piezoelectricity, McGraw-Hill, New York 1946.

[2] J.F. Nye, Physical Properties of Crystals, Oxford Univ. Press, Oxford 1957.

[3] R.M. Martin, Phys. Rev. B 5, 1607 (1972).

[4] S. de Gironcoli, S. Baroni, R. Resta, Phys. Rev. Lett. 62, 2853 (1989).

[5] D.L. Smith, Solid State Commun. 57, 919 (1986); D.L. Smith, C. Mailhiot, Rev. Mod. Phys. 62, 173 (1990) and refs. therein.

[6] L. Kleinman, Phys. Rev. 128, 2614 (1962).

[7] M. Cardona, K. Kunc, R.M. Martin, Solid State Commun. 44, 1205 (1982).

[8] P.N. Keating, Phys. Rev. 145, 637 (1966).

[9] W.A. Harrison, Electronic Structure and the Properties of Solids, Freeman, San Francisco 1980.

[10] A. Dal Corso, R. Resta, S. Baroni, Phys. Rev. B 47, 16252 (1993).

[11] M.P. Halsall, J. E. Nicholls, J. J. Da vies, P. J. Wright, B. Cockayne, Surf. Sci. 228, $41(1990)$.

[12] B.K. Laurich, K. Elcess, C.G. Fonstad, J.G. Beery, C. Mailhiot, D.L. Smith, Phys. Rev. Lett. 62, 649 (1989).

[13] B.V.Shanabrook, D. Gammon, R. Berefoord, W.I. Wang, R.P. Lea vitt, D.A. Broido, Superlatt. Microstruct. 7, 363 (1990).

[14] R. André, C. Deshayes, J. Cibert, Le Si Dang, K. Saminadayar, S. Tatarenko, Phys. Rev. B 42, 11392 (1990).

[15] J. Cibert, R. André, C. Bodin-Deshayes, G. Feuillet, Le Si Dang, K. Saminadayar, S. Tatarenko, J. Cryst. Growth 117, 424 (1992).

[16] D.M. Symons, M. Lakrimi, R.J. Warburton, R.J. Nicholas, N.J. Mason, P.J. Walker, M.I. Eremets, G. Hill, Phys. Rev. B 49, 16614 (1994).

[17] E.A. Caridi, T.Y. Chang, K.W. Goossen, L.F. Eastman, Appl. Phys. Lett. 56, 659 (1990).

[18] J.L. Sánchez-Rojas, A. Sacedón, F. González-Sanz, E. Calleja, E. Muñoz, Appl. Phys. Lett. 65, 2042 (1994).

[19] R. André, PhD. Thesis, Université Joseph Fourier-Grenoble, 1994.

[20] E. Anastassakis, Phys. Rev. B 46, 4744 (1992); J. Appl. Phys. 68, 4561 (1990); Solid State Commun. 84, 47 (1992).

[21] R. André, C. Bodin, J. Cibert, G. Feuillet, Le Si Dang, J. Phys. (France) IV C5, 429 (1993). 
[22] J. Cibert, R. André, C. Bodin, G. Feuillet, P.H. Jouneau, Le Si Dang, Phys. Scr. 49, 487 (1993).

[23] R. André, J. Cibert, Le Si Dang, J. Zeman, M. Zigone, to be published.

[24] K. Strössner, S. Ves, W. Dieterich, W. Gebhart, M. Cardona, Solid State Commun. 56, 563 (1985).

[25] Le Si Dang, H. Okomura, C. Bodin, J. Cibert, G. Feuillet, P.H. Jouneau, Physica $B 185,490$ (1993).

[26] R. André, J. Cibert, Le Si Dang, accepted to Phys. Rev. B.

[27] F. Bassani, K. Kheng, M. Mamor, R.T. Cox, N. Magnéa, K. Saminadayar, S. Tatarenko, J. Cryst. Growth 138, 607 (1994).

[28] X. Li, F. Longenbach, W.I. Wang, Appl. Phys. Lett. 60, 1513 (1992).

[29] C. Bodin, R. André, J. Cibert, Le Si Dang, D. Bellet, G. Feuillet, P.H. Jouneau, Phys. Rev. B 51, 13181 (1995).

[30] W. Langbein, H. Kalt, M. Hetterich, M. Grün, C. Klingshirn, J. Cryst. Growth 138, 191 (1994).

[31] A.N. Cartwright, D.S. McCallum, T.F. Boggess, A.L. Smirl, T.S. Moise, L.J. Guido, R.C. Barker, B.S. Wherrett, J. Appl. Phys. 73, 7767 (1993).

[32] E. Vanelle, D. Block, R. Romestain, J. Cibert, to be published. 